\title{
GENERATING DYNAMIC 3D SCENES FOR RENDERING BENCHMARKS
}

\author{
Vyacheslav Gonakhchyan, Oleg Tarlapan and Vitaly Semenov \\ Ivannikov Institute for System Programming of the Russian Academy of Sciences \\ 109004, Moscow, Alexander Solzhenitsyn st., 25, Russia
}

\begin{abstract}
Performance comparison of different rendering methods is either based on synthetic 3D scenes or commonly used 3D scenes. A lot of static scenes exist but dynamic scenes are not common. Performance of modern rendering methods depends on the dynamic state of objects and varies with an increasing number of objects, degree of scene volatility. In this paper, two new methods of dynamic scene generation are proposed. We consider the bottlenecks of common rendering methods, based on which the parameters of the proposed methods are selected. We propose a method for dynamic scene generation, which allows to create scenes with a specified number of objects, vertices, different appearance order, temporal coherence of visibility, degree of spatial coherence of appearance and other parameters. We propose a method for converting of static scenes into dynamic scenes using space-filling curves to determine the order in which objects appear in a scene. The proposed methods will fill the gap in the field of rendering benchmarks and will allow to use the proven static scenes when evaluating the performance of methods with dynamic objects.
\end{abstract}

\section{KEYWORDS}

Test set Generation, Dynamic 3D Scene, Space-filling Curve, Spatial Coherence

\section{INTRODUCTION}

Capabilities of GPUs are constantly evolving, which allows for rendering of scenes with an increasing number of polygons and objects. Existing methods usually use static scenes and do not take into account the characteristics of scenes that are important for rendering: gradual appearance of new objects, spatial coherence of the appearance in time, the order of object appearance (Bittner et al., 2004; Guthe et al., 2006). We propose a new method for dynamic 3D scene generation with a large number of parameters that allows to vary the computational load on various subtasks of rendering methods.

Rendering of large 3D scenes with dynamic behavior of objects is used in many applications. Although a lot of static 3D scenes exist that are commonly used for performance evaluation, there are no common dynamic 3D scenes. In this paper, we propose a method to convert static scenes to dynamic scenes, where object visibility is determined based on space-filling curves.

Let's consider common static scenes that are used as benchmarks. Following datasets are widely used when testing mesh simplification, mesh compression, surface restoration, and rendering methods (Turk, G. and Mullins, B., 2003; The Stanford 3D Scanning Repository, 2014). Proposed models have a large number of polygons, however, rendering tasks usually also have individual objects with different materials. Following datasets have a large number of different objects, which makes them more suitable for rendering tests, but they consist of static scenes only (Power Plant Model, 2001; Havran, V. et al., 2010; SPECviewperf 13, 2018).

Let's consider existing approaches to performance evaluation when dealing with dynamic objects. Objects can be moved along a path that causes the rebuilding of a hierarchy (Sudarsky \& Gotsman, 1999). In some papers animated data was created using specialized software like Poser and DAZStudio (Wald et al., 2007). Lext et al. (2001) proposed benchmarking datasets and a method for ray-tracing performance evaluation when rendering animated scenes. However, authors have not considered methods of scene generation with a large number of primitives, temporal and spatial coherence. 
Let's consider the space-filling curves in two-dimensional space. Given a square grid with $\mathrm{N}^{\wedge} 2$ cells, space-filling curve is a numbering of grid cells, in which numbers vary from 1 to $\mathrm{N}^{\wedge} 2$. In the limit $\mathrm{N} \rightarrow \infty$, the curve passes through each point of a given region of space. Described properties allow space-filling curves to be used for indexing of complex n-dimensional data, including the clustering of objects in 3D scenes (Lawder \& King, 2000; Pantaleoni \& Luebke, 2010). The most common space-filling curves are Hilbert curve, Morton curve (z-order curve), Row-prime order (see figure 1). In this paper, space-filling curves are used for clustering and calculation of appearance time of resulting objects.
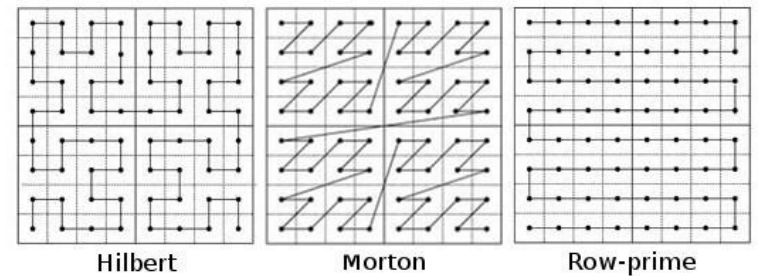

Figure 1. Two-dimensional space-filling curves

\section{DYNAMIC SCENE GENERATION}

Let's consider the bottlenecks in the work of common computer graphics methods to derive the main parameters of proposed methods of dynamic scene generation.

Traditional rendering of polygonal scenes performs the following steps: command recording and submission, vertex transformation, rasterization, fragment shading, blending and output to frame buffer. Each of them might be a bottleneck. To evaluate the performance of command recording and submission, we need to create a large number of objects (entities requiring different pipeline state). To evaluate the performance of vertex transformation, we need a large number of vertices. To evaluate the performance of fragment shader, we need a large number of overlapping surfaces, which generate a lot of pixels on the screen, or expensive shader calculations. The dynamic behavior of objects might cause command buffer overwriting, hierarchy rebuilding. So, the following parameters are required to evaluate the rendering performance: the number of objects in the scene, the number of vertices, the number of overlapping surfaces.

Many rendering methods assume that during animation, when going from frame to frame many objects remain visible (temporal coherence of visibility) (Bittner et al., 2004; Guthe et al., 2006). As a result, visibility calculations can be performed once per multiple frames. In practice, coherence of visibility is achieved when objects are located inside containers (buildings). To generate such scenes, we need to build exterior first and then fill the interior. In order to do that, we identify relatively large objects in a scene. A large object is an object with a diagonal value that is much higher than the average value in a scene. We propose a parameter to output large objects first by traversing the set of objects multiple times until large objects remain in the scene. We also propose a parameter to output small objects first to stress test methods that perform a lot of computations when new objects appear inside view.

In dynamic scenes the order of appearance of objects affects the performance of hierarchical LOD generation. If neighboring objects appear at the same time, then methods can use less levels of detail and reduce the amount of consumed memory. We propose a parameter specifying the degree of spatial coherence of the displayed objects. The parameter ranges from 0 (random appearance of objects) to 1 (appearance of objects along a space-filling curve).

Let's consider the parameters that determine the order of appearance of objects: grid size, traversal order, time interval, space-filling curve. Grid size specifies the granularity of appearance in time, where small size can be used to increase the number of objects appearing at once. We use the right-handed coordinate system, where $\mathrm{Z}$ axis is directed upwards. Traversal order allows to change the direction of traversal for each axis (e.g. XYZ is bottom-up, $\mathrm{XY}-\mathrm{Z}$ is top-down traversal order). Each cell of the grid contains a set of objects, which become visible at time $\mathrm{n} * \Delta \mathrm{t}$, where $\mathrm{n}$ is the cell number, $\Delta \mathrm{t}$ is time interval. We use a space-filling curve (Hilbert, Morton, Row-prime) and traversal order to calculate $n$.

We propose a method for dynamic scene generation that is based on the described parameters. The method creates the file in FBX format with polygonal objects and visibility information. It creates a scene 
with different number of objects and vertices, spatial dimensions, degree of coherence. It can generate a square block of buildings with a given number of buildings with specified number of floors, windows, additional objects per floor, distance between the buildings (see figure 2). It allows to evaluate the performance with a large number of overlapping surfaces. When the number of additional objects is set, it positions them inside the buildings according to the specified traversal order (see figure 3 ). When visibility coherence is set, it displays large objects (walls, slabs) along a curve in several iterations, and then places additional objects inside the buildings (see figure 4). When a space-filling curve is set, it displays the objects in time calculated from the specified curve (see figure 5). When a degree of spatial coherence is set, it displays the objects with the given degree of randomness along the space-filling curve (see figure 6).

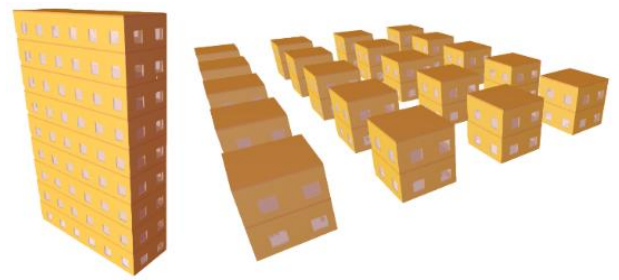

Figure 2. One tall building (left). Block of small buildings (right)
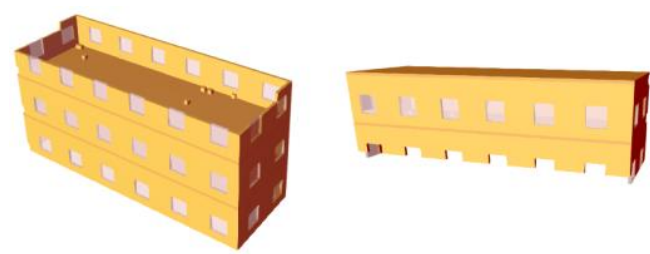

Figure 3. Bottom-up traversal order (left), top-down traversal order (right)

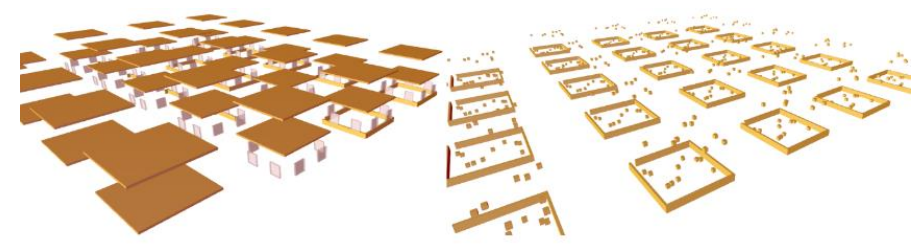

Figure 4. Big objects appearing first (left), small objects appearing first (right)

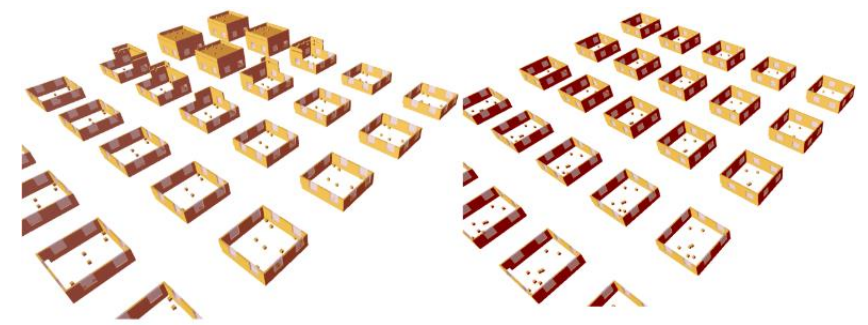

Figure 5. Objects appearing according to Hilbert curve (left) and row-prime order (right). Shown for the same moment of time

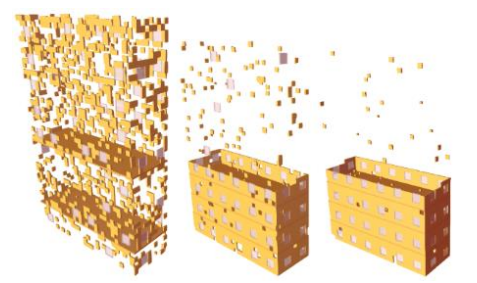

Figure 6. Objects appearing with increasing spatial coherence $(0.1,0.95,0.99)$ along the row-prime curve. Shown for the same moment of time 
We propose a method that converts static scene to dynamic scene, which takes as input a file in FBX format. As output, the method creates a file in FBX format with original objects and visibility information. The method uses all the described parameters except the parameters that specify additional objects. Figure 7 shows an example where a large static scene is converted to the dynamic scene by displaying objects in row-prime order.
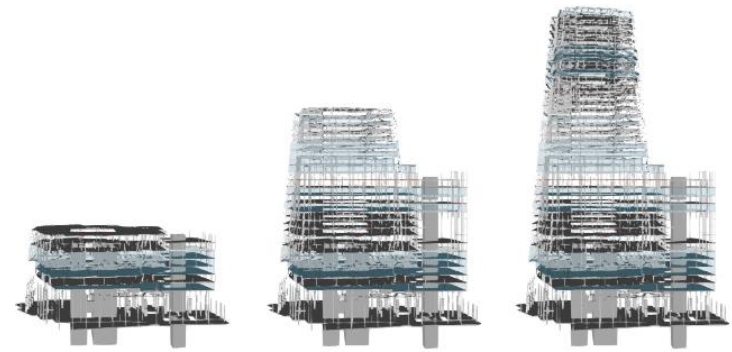

Figure 7. Generation of the dynamic scene from the static scene. Objects gradually appear along the row-prime curve

\section{CONCLUSION}

We developed two methods of dynamic scene generation. The first method generates a block of buildings with a given number of buildings, additional objects, temporal coherence of visibility, spatial coherence of appearance. It can be used for performance benchmarking of different rendering methods with an increasing number of objects. The second method takes a static 3D scene and converts it into a dynamic 3D scene, using space-filling curves to determine the order of appearance of objects. It allows to create dynamic scenes from widespread static scenes.

\section{REFERENCES}

Bittner, J. et al., 2004. Coherent hierarchical culling: Hardware occlusion queries made useful. In Computer Graphics Forum, Vol. 23, No. 3, pp. 615-624.

Guthe, M. et al., 2006. Near Optimal Hierarchical Culling: Performance Driven Use of Hardware Occlusion Queries. In Rendering Techniques, pp. 207-214.

Havran, V. et al., 2010. MPI Informatics Building Model as Data for Your Research (online). Available online: http://resources.mpi-inf.mpg.de/mpimodel/v1.0/

Lawder, J. K. and King, P. J., 2000. Using space-filling curves for multi-dimensional indexing. In British National Conference on Databases, pp. 20-35.

Lext, J. et al., 2001. A benchmark for animated ray tracing. IEEE Computer Graphics and Applications, Vol. 21, Issue 2, pp. 22-31.

Pantaleoni, J. and Luebke, D., 2010. HLBVH: hierarchical LBVH construction for real-time ray tracing of dynamic geometry. In Proceedings of the Conference on High Performance Graphics, pp. 87-95.

Power Plant Model, 2001. Available online: http://gamma.cs.unc.edu/POWERPLANT/

SPECviewperf 13, 2018. Available online: https://www.spec.org/gwpg/gpc.static/vp13info.html

Sudarsky, O. and Gotsman, C., 1999. Dynamic scene occlusion culling. IEEE Transactions on Visualization and Computer Graphics, Vol. 5, Issue 1, pp. 13-29.

The Stanford 3D Scanning Repository, 2014. Available online: http://graphics.stanford.edu/data/3Dscanrep/

Turk, G. and Mullins, B., 2003. Large Geometric Models Archive. Available online: https://www.cc.gatech.edu/projects/large_models/

Wald, I. et al., 2007. Ray tracing deformable scenes using dynamic bounding volume hierarchies. ACM Transactions on Graphics (TOG), Vol. 26, Issue 1, Article 6. 